\title{
The Respective Roles of Controlled Clinical Trials and Cohort Monitoring Studies in the Pre- and Postmarketing Assessment of Drugs
}

\author{
Muriel Vray, ${ }^{1}$ Bernard Hamelin, ${ }^{2}$ Patrice Jaillon ${ }^{3}$ and the Participants in Round Table No. 2, \\ Giens $X X^{*}$ \\ 1 Unité d'Epidémiologie des Maladies Emergentes, Institut Pasteur, Paris, France \\ 2 Laboratoires AstraZeneca, Rueil-Malmaison, France \\ 3 Hôpital Saint-Antoine, Paris, France
}

\begin{abstract}
The respective roles of controlled clinical trials and observational studies (cohort or case-control studies) in evaluating the efficacy, safety and usefulness of a drug were analysed. A randomised, controlled, double-blind study is the best method of estimating the efficacy of a treatment. It provides the least biased and most robust estimate of the causal relationship. In certain situations and on the basis of certain criteria, observational studies can have a proof-of-efficacy value. Randomised, controlled, pre- and postmarketing authorisation (MA) clinical studies identify the rarer adverse effects and compare them with those resulting from the reference treatment. Before the MA, the pooled safety data from different controlled trials can provide an estimation of relatively frequent adverse events and subjects at risk. However, an observational study is the most appropriate method of evaluating the safety of a drug in the currently used conditions. By definition, a drug influences the health of a population if it directly or indirectly improves its health. A drug would have a major role in public health if it reduced mortality or morbidity related to a particular disease or if it improved the quality of life of patients with this disease. Prior to marketing a product, modelling is the approach of choice to quantify the expected effect. Pragmatic, postmarketing trials and observational studies are the reference methods used to define the population affected, the efficacy and safety of the drug in a real situation and its usefulness for public health. In conclusion, randomised clinical trials remain the reference approach for evaluating efficacy, while observational studies have a confirmatory value. Observational studies are the most appropriate way of evaluating safety in the currently used conditions, as the clinical trial has limited indications. In the interests of public health, modelling is the pre-marketing approach of choice, while pragmatic trials and observational studies are the postmarketing reference approaches.
\end{abstract}

Keywords: methodology, controlled trials, cohorts, pre- and postmarketing

To date, the assessment of the benefit/risk ratio of new drugs has been mainly based on the findings of the controlled clinical trials required to obtain a marketing authorisation (MA) for medicinal products. After the MA has been obtained, and apart from occasional pharmacoepidemiological studies of cohorts or casecontrol studies, often carried out on behalf of the pharmaceutical company, the follow-up of medicinal products has consisted of the notification of adverse events by healthcare professionals to the national pharmacovigilance system or to the pharmacovigilance department of the company involved. The French Medicines
Agency, Afssaps (Agence française de sécurité sanitaire des produits de santé), can also decide to initiate national pharmacovigilance surveys with a follow-up targeting the objectives of safety and public health. In contrast, the concepts underlying the definition of the 'population affected' by the medicinal product or of confirming the quantitative therapeutic effect produced by the medicinal product in 'real life', i.e. after it has been placed on the market, have rarely been assessed.

According to the French General Directorate for Health (Direction Générale de la Santé), the requirement to investigate the

\footnotetext{
* For a list of participants, please see the end of the article.
} 
efficacy and safety of a new medicinal product after it has been placed on the market has become scientifically obvious, even if it is not yet a regulatory requirement. The recent withdrawal of a nonsteroidal cyclo-oxygenase (COX)-2 antagonist, rofecoxib, after it displayed cardiovascular toxicity has demonstrated the need for post-MA data. It is now beyond question that the populations of patients treated with a new drug sometimes differ profoundly from the populations of patients included in the pre-MA clinical trials, and that the efficacy/safety data for the drug reported before the MA cannot therefore be directly transposed to the use of the drug after the MA has been obtained.

It is therefore quite correct that the methods suitable for use both before and after the MA to determine the real benefit/risk ratio of a new drug should be analysed and compared. The objective of this Round Table was to discuss the respective roles of controlled clinical trials and cohort monitoring in the pre- and post-MA assessment of drugs.

\section{Assessment of Efficacy}

\subsection{Trials and Studies}

Randomised controlled trials (RCT) carried out under doubleblind conditions remain the best method of determining the efficacy of a drug. The random selection of the treatments allocated to the patients and maintenance of the double-blind conditions during the trial are the best 'defences' against the possibility of bias that would distort the findings of the trial (bias in selecting the patients or in allocating the treatments, subjectivity in interpreting the results etc.). The randomised double-blind clinical trial makes it possible to obtain the least biased and most robust evidence of the efficacy of the drug. Such trials provide the highest level of proof of the efficacy of a drug, and constitute the essential methodological basis of pre-MA phase III clinical trials, which are used to define the therapeutic indication of the drug, the target population, the dosage to be administered and any precautions or contra-indications that should be imposed after the MA.

Observational studies of cohorts, retrospective or prospective case-control studies, open trials of effectiveness, and those using random selection must also be included in the assessment of the efficacy of a drug. These various types of clinical studies, so far restricted to the post-MA period, could, under certain conditions, provide additional evidence for the efficacy of the drug.

\subsection{Quality Criteria}

Trials such as randomised, controlled trials and observation studies can only be used to estimate the efficacy of a drug if they are performed according to certain criteria:

- The appropriate methodology must be chosen if the findings are to be extrapolated to the population of patients. Possible sources of bias must be identified, and then taken into account when analysing and interpreting the data. The choice of the comparator drug must be pharmacologically justified.

- In controlled trials, a test designed to demonstrate the drug's superiority over a reference drug provides better evidence for efficacy than a test to demonstrate that it is not inferior to the reference agent. If the drug is shown to be not inferior, efficacy is demonstrated only indirectly versus a reference drug. Non-inferiority, and therefore efficacy, is established relative to an arbitrarily defined marker of non-inferiority.

- Good practice in carrying out the trials or observational studies, the size of the confidence interval, and the robustness of the findings are all important factors for consideration in validating the findings.

- The extent of the difference observed in a controlled trial is a crucial factor when evaluating the pertinence of the findings of the trial. We know, for instance, that simply determining the reduction of the relative risk in a controlled trial can mislead clinicians, who do not always realise that assessing the reduction of the absolute risk considerably diminishes the real value of the new drug compared with that of the reference drug.

- Finally, the reproducibility of the data obtained in the various studies can also be taken as an indication of safety if the results are convergent.

\subsection{Respective Roles of Controlled Trials and Observational Studies}

Randomised, double-blind controlled clinical trials provide the highest level of proof of the therapeutic efficacy of a drug. It is unusual for a single cohort observational study to establish a sufficient level of proof to validate the registration of a drug or a new indication. The Committee for Proprietary Medicinal Products (CPMP) has stipulated the criteria required to justify the impossibility of carrying out a controlled trial, in the light of current knowledge:

- a serious illness with little or no likelihood of spontaneous improvement;

- exceptional efficacy with clear individual outcomes;

- a documented and acceptable safety profile;

- no alternative treatment available; 
- the search for data from previous trials.

In the case of a drug with a rare indication, after carrying out a controlled trial it may be useful to set up a cohort study, e.g., when assessing the efficacy of an antibiotic in a disorder caused by multiresistant pathogens following the assessment of its efficacy in an infectious disease involving more commonly encountered pathogens.

An RCT may be accompanied by a non-controlled observational study targeting, for instance, patients who were not classified as eligible for the trial on the basis of the non-inclusion criteria concerning the seriousness of the disease.

A cohort, observational study or a retrospective case-control study can identify a useful effect (efficacy or safety) of the drug that could be subsequently confirmed by an RCT.

Finally, and this is the most common situation, an RCT can be followed by a cohort observational study or an effectiveness trial in order to confirm the efficacy of the drug during long-term follow-up and under real conditions of use.

\section{Assessment of Safety}

\subsection{Before the Marketing Authorisation (MA), the Clinical Trial is the Reference Method for Assessing Safety}

Randomised, controlled clinical trials provide the most robust demonstration of a cause and effect relationship, as well as a comparison between the treatment being investigated and the comparator drugs, in particular for adverse events classified as 'frequent' (>1\%) or previously identified. However, it does have some important limitations:

- The study design is experimental, because the treatments are randomly allocated, and so does not take prescriber or patient preference into account. This means that a clinical trial provides a rather poor reflection of the complexity of clinical situations.

- The eligibility criteria for trials are often rather restrictive in order to ensure that the population assessed is as homogeneous as possible. This means that the findings obtained from trials cannot always be extrapolated to the general population, notably with regard to safety.

- The clinical trial often lacks the statistical power to detect rare $(<1 / 1000)$ or very rare $(<1 / 10000)$ adverse events.

The safety data reported during pre-MA trials are pooled in order to examine safety assessments in terms of the pharmacological profile of the drug and the various categories of patients (demographic characteristics, previous history, etc.).
Pooling data from different trials increases statistical power, and this makes it possible to carry out analyses of subgroups of high-risk patients. However, these methods of analysis do not always allow rare events to be detected. If a signal is detected during the initial evaluation of pooled data, it is important to initiate either clinical trials in the particular subgroups identified as being at high risk or case-control observational studies (statistically more powerful, but more liable to various forms of bias) in the post-MA assessment in order to confirm or refute the possibility of a potential risk to the patient.

\subsection{During Post-MA Assessment, Pharmacovigilance and Pharmacoepidemiological Studies are Used to Complete the Initial Assessment}

Recent experience has shown that the potential risks of a drug may not become clear until after the MA has been obtained, as a result of either identifying a series of cases during post-MA monitoring of adverse events (pharmacovigilance), or by setting up specific post-MA studies (usually of a pharmacoepidemiological nature).

\subsubsection{Pharmacovigilance Data}

Pharmacovigilance data make it possible to check the safety of drugs under real conditions of use, and to detect any increase in the risk of serious adverse events. However, these data are not suitable for estimating the incidence in the population exposed to the treatment (the population treated). They raise the question of the variable quality of the information gathered and the way this information is reported. This system is based on identifying groups of cases, and is a very efficient way of detecting a warning signal.

\subsubsection{Observational Studies}

Observational studies differ from controlled clinical trials in that the treatment assigned to the subject has been deliberately selected by the physician. They make it possible to assess the safety of the drug, 'in real life', within the target population and under the conditions in which it will be used by patients. Two different types of observational studies can be distinguished:

- 'Cohort' studies, which measure the frequency of an event, and determine whether there is any association between an exposure factor (the drug) and the event (the adverse event).

- Case-control studies, which determine only whether there is any association.

The risks of bias associated with observational studies carried out to assess the effects of a treatment have been clearly described in medical literature. The findings are somewhat limited, because of the existence of unidentified prognostic factors. 
However, by simulating routine clinical conditions as closely as possible, these studies make it possible to better extrapolate the results to the entire population treated in real life; in addition, thanks to their greater statistical power, which results from the often very large sample populations involved, studies of this type are more suitable than spontaneous reporting or controlled trials for identifying and measuring rare or very rare adverse events.

\section{Measurement of the Public Health Effect}

After a drug has demonstrated that its benefit/risk ratio is sufficient to obtain an MA, a population-type assessment is necessary to determine its public health value.

By definition, a drug influences the health of a population if it can directly or indirectly improve the health status of this population. The public health effect can be defined as the number of morbid events or handicaps avoided in a given population over a specific period of time.

A drug will be of major public health interest if it reduces the mortality or morbidity linked to an illness, or if it improves the quality of life of the patients with this illness.

\section{1 Pre-MA Context}

Before the drug is marketed, the questions raised concern the prevalence and incidence of the illness on the basis of various levels of gravity.

The next step consists of identifying the ideal target population, i.e. the population that should theoretically be able to benefit from using the drug, and the population likely to actually use it in practice. It is also necessary to identify the population that will benefit most from the drug while taking into account the practical realities of healthcare.

The expected impact for public health can be estimated on the basis of these factors and the quantitative effect measured in the studies used to compile the MA file.

Together with the findings from controlled, randomised and if possible double-blind phase II/III trials in the MA file, the data that will make it possible to evaluate the target population are often obtained by descriptive studies carried out on cohorts or from panels for the other drugs in the class.

Ad hoc observation-type studies based on representative samples can provide answers to the questions of how to define the disease prevalence and target populations.

It is not possible to determine the extent of the effect in the real situation of patient care on the basis of morbidity/mortality criteria, which are usually not documented, except in the case of an evaluation that, in real life, can be fully extrapolated to find- ings reported during pre-MA trials. Mathematical models can, by simulation, quantify the expected effect. To do this, we need to understand the model of the effect of the drug - the relationship between the frequency of the clinical event during treatment and frequency without treatment, and which is specific to each drug, disease and therapeutic objective - provided that certain criteria are verified: clinical criterion available in phase III studies (and not only the substitution criterion), access to all the data and description of the algorithm used.

Unfortunately, we do not usually have this information when a drug is put on the market. This means that we have to try to assess the model on the basis of the results of the trial by reasoning in terms of therapeutic class, which is obviously not possible in the case of a drug belonging to a new therapeutic class. Predicting the effect, on the basis of these models, will be more reliable if it is based on trials in which the populations are heterogeneous and reflect the real situation in practice. The value of such models is evident in the analyses of sensitivity which, by varying the parameters, make it possible to quantify the effects. However, even if these models are acceptable at the time the drug is registered, they cannot be used by the sponsors to avoid performing real life studies to determine its effectiveness.

Depending on the disease concerned, the drugs, and the way the patients are managed, several different methods can be considered.

\subsection{Post-MA Context}

Once a drug is on the market, many questions remain to be answered, such as its efficacy and safety under practical prescribing conditions, and also questions relating to the habits of patients and of prescribers.

The identification of the population actually treated, i.e. which actually receives treatment with the drug, and which may differ from the target population specified in the official indications for this drug, is a major question in public health.

Studies intended to assess the proper use of the drug, its impact on the way a disease is managed, or the identification of factors predictive of a good response or an acceptable level of safety should also be initiated.

To answer these various questions, we have to choose between an observational approach and an experimental approach such as a pragmatic trial.

In an observational approach, comparing cohorts of subjects treated with different drugs, a population or a phenomenon is simply observed without influencing, or influencing as little as possible, the natural course of events. This suggests that these studies will not distort reality too much. In fact, it is illusory to 
imagine that a study will have no effect at all on the behaviour of the patients and the doctors when they know they are being observed. In this context of observational studies, one may also wonder whether the prospective or retrospective approach should be taken to record information. A prospective approach, in which the subjects treated are kept under observation until the event being investigated occurs, should apparently result in fewer missing data. However, it may have more influence on the reality of patient management. On the other hand, in a retrospective approach, the effect (or exposure) being investigated will be subject to greater recall bias, resulting in an over-estimation of the exposure of the cases.

The findings of these observational studies should take into account the bias resulting from the degree of representativeness of the sample studied, the confounding factors and the often large number of subjects lost to follow-up. Analyses that include adjustment for the confounding factors should be performed.

In contrast, a more experimental approach would involve carrying out a clinical trial in which the drugs or treatment strategies are allocated randomly. This option would appear attractive, because it seems to make it possible to 'control' the noncomparability bias of the group at inclusion but, in fact, does not exactly represent reality, even though the inclusion criteria for the patients, the monitoring and the assessment criteria, are much closer to 'real life', and differ from those used for phase III trials. Once again, there is a risk of modifying the behaviour of both physicians and patients who know they are being 'monitored'. We can also suppose that some patients/physicians will refuse to take part in an experiment in which the patients will be assigned a treatment on a random basis, even if there is no scientific evidence in favour of their being treated specifically by one or other of the treatments being investigated.

The estimation of usefulness for public health is therefore based mainly on cohort studies or effectiveness studies.

\section{Conclusion}

Randomised clinical trials remain the reference method for assessing efficacy, and observational studies are of complementary value. In some situations, and given that certain criteria are verified, observational studies can be used to demonstrate efficacy.

Observational studies are more suitable for assessing safety under real conditions of use, and clinical trials have limited indications. The results of double-blind controlled trials using pooled data can be used to identify relatively frequent events and highrisk subjects.

Modelling is the ideal method for use prior to marketing to assess the usefulness of a drug for public health. The data that constitute the MA file often allow, at best, an estimation of the expected usefulness for public health.

Pragmatic post-MA trials and observational studies are the best approaches for assessing the effect of a drug on the population treated.

\section{Participants}

M. Andréjak (CHU, Amiens), R. Benamouzig (Hôpital Avicenne, Bobigny), J.-P. Boissel (Faculté de Médecine, Lyon), C. Bremard-Oury (Aventis, Paris), C. Brun-Strang (Novartis, Rueil-Malmaison), E. Caulin (Aventis, Paris), D. Cellier (Merck Santé, Lyon), D. Costagliola (INSERM, Paris), V. Daurat (AP-HP DRRC, Paris), A. El Hasnaoui (GSK, Marly le Roi), J.-M. Goehrs (Merck Sharp \& Dohme-Chibret, Paris), B. Hamelin (AstraZeneca, Rueil-Malmaison), P. Jaillon (Hôpital St-Antoine, Paris), C. Jouan-Flahault (LEEM, Paris), J.-L. Montastruc (Faculté de Médecine, Toulouse), N. Moore (Hôpital Pellegrin, Bordeaux), A. Puech (Sanofi Synthelabo, Chilly-Mazarin), M. Ricatte (CNAMTS, Paris), P. Ricordeau (CNAMTS, Paris), M.-H. Rodde-Dunet (CNAMTS, Paris), E. Rouffiac (Abbott, Rungis), T. Simon (Hôpital St-Antoine, Paris), P. Simon (Sanofi Recherche, Paris), A. Spriet (Spriet Conseil, Paris), E. Van Ganse (CHU, Lyon), P. Velicitat (Boehringer-Ingelheim, Reims), M. Vray (Institut Pasteur, Paris).

Correspondence and offprints: Bernard Hamelin, Laboratoire AstraZeneca, 1 place Renault, 92844 Rueil-Malmaison Cedex, France.

E-mail: bernard.hamelin@astrazeneca.com 